\title{
COMUNICAÇÃO CIENTÍFICA
}

\section{CARGA ÓTIMA DE FRUTOS NA MACIEIRA 'PRINCESA' DE BAIXO REQUERIMENTO EM FRIO INVERNAL: RESULTADOS PRELIMINARES ${ }^{1}$}

\author{
DAMIÁN CÉSAR CASTRO ${ }^{2}$, NORMA MICHELOUD ${ }^{3}$, MARCELA BUYATTI ${ }^{4}$, \\ NORBERTO FRANCISCO GARIGLIO ${ }^{5}$
}

RESUMO - O objetivo do trabalho foi avaliar o efeito da carga de frutos sobre a produção total, calibre, massa média, comprimento dos ramos e intensidade da frutificação no ano seguinte, em plantas de macieira (Malus $\times$ domestica Borkh.) cv. 'Princesa'. Durante os anos de 2009 e 2010, as infrutescências foram submetidas a raleio manual, deixando nas árvores uma carga de 1 a 9 frutos por centímetro quadrado de superfície do tronco (FCQ). A produção total aumentou com o incremento da carga frutífera desde 1 a 9 FCQ, enquanto a massa média dos frutos diminuiu no mesmo intervalo. A quantidade de frutos pequenos aumentou até atingir 10\% do total nas plantas de maior carga e a proporção de frutos de tamanho médio cresceu, alcançando $35 \%$ do total da produção. No entanto, a quantidade de frutos grandes não foi afetada pelo nível de carga, mantendo-se em maior proporção com relação ao total colhido ( $\approx 66 \%)$. O crescimento dos ramos, até 60 dias após a colheita, esteve negativamente afetado pela intensidade de carga. Observouse que não houve alternância de safra no ano seguinte ao experimento. A carga ótima para minimizar a produção de frutos pequenos, alcançando a maior produção total sem debilitar as árvores, pode-se atingir no intervalo entre 5 e 9 FCQ.

Termos para indexação: raleio manual, qualidade de frutos, crescimento vegetativo, Malus domestica.

\section{OPTIMUM CROP LOAD FOR LOW-CHILL APPLE cV PRINCESA: PRELIMINARY RESULTS}

\begin{abstract}
The aim of this study was to evaluate the effect of crop load on yield, fruit size, vegetative growth and return to bloom of low-chill apple (Malus $\times$ domestica Borkh.), cv. 'Princesa'. During 2009 and 2010 crop load was manually adjusted from 1 to 9 fruits per unit of trunk cross-sectional area (TCSA). Fruit yield was positively related to crop load, but mean fruit weight decreased with increasing crop load. The proportion of small and middle-sized fruits increased with rising crop load reaching $10 \%$ and $35 \%$ respectively of the total yield at the highest crop load. However the proportion of large-sized fruits did not change with the increase in crop load. Shoot length at 60 days after harvest was negatively related to crop load whereas return to bloom was not affected. These results demonstrates that the optimum crop load for 'Princesa' apple ranges between 5 to 9 fruits per unit of TCSA.

Index terms: Malus domestica, hand thinning, trunk cross-sectional area, fruit quality, vegetative growth.
\end{abstract}

${ }^{1}$ (Trabalho 088-14). Recebido em: 28-02-2014. Aceito para publicação em: 27-02-2015.

${ }^{2}$ DSc. Professor de Fruticultura da Facultad de Ciencias Agrárias da Universidad Nacional del Litoral. Argentina. Kreder 2805 (3080) Esperanza, SFe Argentina. Telefone: +54 (3496) 426400. E-mail: dcastro@fca.unl.edu.ar

${ }^{3}$ MSc. Professora de Fisiologia da Facultad de Ciencias Agrárias da Universidad Nacional del Litoral. Argentina. E-mail: nmicheloud@ fca.unl.edu.ar

${ }^{4}$ MSc. Professora de Floricultura da Facultad de Ciencias Agrárias da Universidad Nacional del Litoral. Argentina. E-mail: mbuyatti@ fca.unl.edu.ar

${ }^{5}$ DSc. Professor de Fruticultura da Facultad de Ciencias Agrárias da Universidad Nacional del Litoral. Argentina. E-mail: ngarigli@ fca.unl.edu.ar 
O desenvolvimento de novas cultivares e tecnologias de cultivo tornaram possível a expansão exitosa do plantio de macieira em locais com baixa disponibilidade de frio invernal (O'ROURKE, 2003). Atualmente, existem pelo menos 48 cultivares e seleções de macieiras de baixos requerimentos de frio que permitem seu cultivo em zonas com invernos amenos no mundo todo (HAUAGGE; CUMMINS, 2000). No continente americano, uma das cultivares mais difundidas é a 'Princesa', utilizada em regiões de menor acúmulo de frio da Argentina (ALAYÓN LUACES et al., 2004; SEIPEL et al., 2009), Brasil (HAUAGGE; CUMMINS, 2000) e México (MENDOZA-GONZÁLEZ et al., 2008). Embora existam estudos realizados sobre o comportamento produtivo (LOPES et al., 2013), qualidade do fruto (CHAGAS et al., 2012) e a aptidão como polinizadora (ALBUQUERQUE JR. et al., 2010) desta cultivar, não existem ainda informações suficientes em relação ao manejo da carga de frutos. A intensidade da carga de frutos tem grande efeito sobre atributos como: massa ou calibre, cor da casca, firmeza da polpa e o conteúdo de sólidos solúveis totais dos frutos (IGLESIAS et al., 2008; 2012). Dada a importância da intensidade de carga sobre a produção e a qualidade comercial da fruta produzida, existem numerosas pesquisas que objetivaram determinar a carga ótima de frutos por planta em diferentes cultivares (EMBREE et al., 2007; LINK, 2000; TREDER, 2008; 2010; VOLZ, 1988; WRIGHT et al., 2006). Porém, esta carga ótima é diferente entre cultivares de macieira, variando de três até nove frutos por centímetro quadrado de superfície transversal do tronco (FCQ) (EMBREE et al., 2007; TREDER, 2008; 2010; WRIGHT et al., 2006). Atingir uma carga ótima de frutos é de grande importância agronômica para obter alta produção com adequada qualidade comercial, sem debilitar as árvores e sem induzir uma alternância de safra (EMBREE et al., 2007; LINK, 2000). Portanto, a carga ótima de frutos para uma espécie e/ou cultivar é aquela que permite atingir um ponto de equilíbrio entre estes fatores. O objetivo do trabalho foi avaliar a relação entre a quantidade de frutos por unidade de superfície do tronco e a produção total, qualidade e tamanho dos frutos da macieira 'Princesa' com o propósito de estabelecer sua carga ótima.

Realizaram-se dois experimentos, em anos consecutivos (2009 e 2010), com plantas de macieira (Malus $\times$ domestica Borkh), cv. 'Princesa' (Anna $\times$ NJ56), de três anos de idade, enxertadas em porta-enxerto MM 111 em um pomar comercial, em Santa Fe, Argentina (31'32'58'S; 6040'36”O; $18 \mathrm{~m}$ acima do nível do mar). A região tem uma disponibilidade média de frio de $537 \pm 128$ horas com temperaturas $\leq 7,2{ }^{\circ} \mathrm{C}$ (GARCÍA, 2012). A cultivar 'Princesa' necessita de 450 unidades de frio para um adequado comportamento agronômico (HAUAGGE; CUMMINS, 2000). A densidade de plantio foi de $1.250 \mathrm{pl} \cdot \mathrm{ha}^{-1}$ com um espaçamento de $4 \times 2$ m (em direção E-O), conduzidas em suporte tipo 'espaldeira', em sistema de palmeta horizontal, sendo a altura média das plantas de $3 \mathrm{~m}$ aproximadamente. No estádio 72 da escala de desenvolvimento fenológico BBCH (MEIER et al., 1994), que corresponde a frutos com $20 \mathrm{~mm}$ de diâmetro, realizou-se o raleio manual das infrutescências das plantas. Os tratamentos variaram de um a nove frutos por centímetro quadrado de superfície transversal do tronco (FCQ).

$\mathrm{Na}$ colheita, avaliaram-se as seguintes variáveis: massa fresca dos frutos $(\mathrm{g})$; produção total de fruta $\left(\mathrm{kg} \mathrm{pl}^{-1}\right)$; produção de frutos pequenos (diâmetro $<60 \mathrm{~mm}, \mathrm{~kg} \mathrm{pl}^{-1}$ ); produção de frutos médios (diâmetro entre 60 e $70 \mathrm{~mm}$; $\mathrm{kg} \mathrm{pl}^{-1}$ ); produção de frutos grandes (diâmetro $>70 \mathrm{~mm}$; kg $\mathrm{pl}^{-1}$ ) e comprimento médio dos ramos do ano $(\mathrm{cm})$. A produção total avaliou-se através da pesagem do conjunto dos frutos colhidos por planta. A massa fresca média dos frutos e a produção de frutos pequenos, médios e grandes estimaram-se através de uma amostra de 40 frutos por planta. $\mathrm{O}$ comprimento dos ramos registrou-se a partir de uma amostra de 15 ramos por planta, em três momentos diferentes: 60 e 30 dias antes da colheita (60 e 30 DAC) e 60 dias após a colheita (60 DApC). Em cada amostragem, mediram-se, aleatoriamente, ramos em ambos os lados da copa, a 1,5 $\mathrm{m}$ acima do nível do solo (neste caso os ramos puderam ou não coincidir nas diferentes datas de amostragem). Também se registrou a intensidade de frutificação no ano seguinte, contabilizando os frutos totais por planta, antes da queda fisiológica, que ocorreu aproximadamente 35 dias após a plena floração (no mês de outubro). Utilizou-se um delineamento experimental inteiramente casualizado, com cinco repetições por tratamento, sendo cada repetição composta por uma planta. Os dados de qualidade e produção total de frutos submeteram-se ao ajuste de regressões lineares e não lineares, utilizando o software estatístico InfoStat (DI RIENZO et al., 2012). Os dados de comprimento dos ramos e da frutificação do ano seguinte submeteram-se ao ajuste de modelos lineares gerais (MLG), mediante a função lme do pacote nlme (PINHEIRO et al., 2013) da linguagem de programação estatística R (R DEVELOPMENT CORE TEAM, 2013), utilizando a interface provida por InfoStat (DI 
RIENZO et al., 2012). Neste caso, a comparação entre as médias realizou-se através do teste de Di Rienzo, Guzmán e Casanoves (DGC) (DI RIENZO et al., 2002). A avaliação da homogeneidade da variância e a normalidade dos erros efetuaram-se graficamente. Quando a homogeneidade da variância e a normalidade dos erros não foram cumpridas no ajuste dos MLGs, utilizou-se da função varIdent do pacote nlme do R (PINHEIRO et al., 2013) para sua correção. A correlação entre as medidas repetidas do comprimento dos ramos corrigiu-se com da função de simetria, composta (corCompSymm) do pacote nlme do R (PINHEIRO et al., 2013).

A produção total aumentou com o incremento do nível de carga de frutos, ajustando-se a um modelo logarítmico (Tabela 1). Até o nível de $5 \mathrm{FCQ}$, a produção total cresceu aproximadamente $11 \mathrm{~kg} \mathrm{pl}^{-1}$, mas acima desse nível a taxa de aumento foi cada vez menor, chegando ao limite máximo de $25 \mathrm{~kg}$ $\mathrm{pl}^{-1}$ com uma carga frutífera de 9 FCQ (Tabela 1). A carga de frutos também afetou a distribuição dos diâmetros dos frutos nas três categorias avaliadas (Tabela 1). A produção de frutos pequenos aumentou com o incremento da carga, no entanto foi baixa $(<11,6 \%)$ em relação à produção total (Tabela 1). Entretanto, a quantidade de frutos médios teve um grande aumento até o nível de carga de $5 \mathrm{FCQ}$, diminuindo sua taxa de incremento acima deste nível, até chegar ao limite aproximado de $35 \%$ em relação à produção total. A quantidade de frutos grandes não foi afetada pelo nível de carga frutífera $(p>0,05)$ (Tabela 1). No entanto, manteve valores altos, tanto em termos de quilogramas por planta, quanto em termos de porcentagens, variando entre $82,4 \%(8,9$ $\left.\mathrm{kg} \mathrm{pl}^{-1}\right)$ e $56,7 \%\left(12,7 \mathrm{~kg} \mathrm{pl}^{-1}\right)$ da produção total até o nível de 6 FCQ (Tabela 1). Deste modo, o aumento da carga frutífera não causou o aumento da produção de frutos pequenos; ao contrário, ocasionou o aumento da produção de frutos médios. Este comportamento observou-se em cultivares de macieira com a tendência à produção de frutos grandes, como 'Elstar' (LINK, 2000). Por outro lado, observou-se uma tendência logarítmica na evolução da massa fresca média dos frutos, diminuindo seu valor com o aumento do nível de carga de frutos (Tabela 1). A maior massa fresca média dos frutos (197,1 g) atingiu-se com a menor carga. Até o nível de carga de 5 FCQ, a massa fresca dos frutos foi maior do que 150 gramas, confirmando o potencial genético da cv. Princesa para a formação de frutos médios a grandes, tal como foi observado em regiões bem diferentes, como as do centro (SEIPEL, 2009) e do nordeste (ALAYON LUACES, 2004) da Argentina e do Sul do Brasil (CHAGAS et al., 2012). A maior queda na massa fresca média dos frutos observou-se no intervalo de carga de 1 a 4 FCQ $(\approx 40 \mathrm{~g})$. O escasso aumento da massa fresca média dos frutos e a grande diminuição da produção total com o decréscimo na carga frutífera são fatos conhecidos (EMBREE et al., 2007; TREDER, 2008, 2010; VOLZ, 1988). Assim, a produção total é determinada pela quantidade total de frutos e não pela massa fresca média dos mesmos (ELFVING; SCHECHTER, 1993). No entanto, se a carga de frutos for maior do que a carga ótima, haverá perda do valor comercial da produção, devido à maior redução do tamanho dos frutos.

O comprimento dos ramos foi afetado tanto pela carga de frutos quanto pelo progresso do tempo (Figura 1). O aumento da carga de frutos acima de 5 FCQ influiu negativamente no comprimento dos ramos, efeito observado até 60 dias após a colheita. Nas árvores com níveis de carga de frutos maiores do que $5 \mathrm{FCQ}$, a presença de frutos até 30 dias antes da colheita produziu a redução no crescimento dos ramos de aproximadamente $45 \%$ (Figura 1). Nessas plantas, a colheita dos frutos permitiu o incremento no comprimento dos ramos de $68 \%$ ao longo de 60 dias $(24,7 \mathrm{~cm}$ em média 30 DAC vs 41,6 cm em média 60 DapC) (Figura 1). Essa resposta deve-se à dominância dos frutos na partição de fotoasimilados, que aumenta a proporção de matéria seca total dos frutos e restringe o crescimento vegetativo (HO, 1992; INGLESE et al., 2002; MARCELIS, 1996). No entanto, na região onde foram feitas estas pesquisas, a estação de crescimento dos ramos após a colheita foi longa ( $\approx 100$ dias), o que permitiu uma recuperação do crescimento dos ramos das árvores com carga de frutos maior do que $5 \mathrm{FCQ}$, chegando até os $40 \mathrm{~cm}$ aos $60 \mathrm{DapC}$, ainda restando um mês de crescimento. No entanto, esse crescimento foi $34 \%$ menor que o alcançado pelos ramos das árvores com carga de frutos menores do que 5 FCQ (Figura 1). A intensidade da floração e da frutificação do ano seguinte ao experimento não foi afetada pelo nível de carga de frutos $(p>0,05)$ e permaneceu com altos valores em todo o intervalo de carga de frutos (Tabela 1). Apesar da alternância da floração que mostra algumas cultivares (EMBREE et al., 2007; MELAND, 2009), no entanto isso não se observa em todas as cultivares de macieira (DENNIS JR., 2003). Segundo Link (2000), a quantidade ótima de frutos de uma árvore frutífera deveria maximizar a quantidade de frutos grandes e minimizar a quantidade de frutos pequenos, atingindo ademais a maior produção total. Igualmente, esse nível de carga frutífera não deve produzir uma bianualidade na produção de frutos e tampouco debilitar as árvores (EMBREE et al., 2007). Os dados desta pesquisa demonstram que 
existe o umbral de carga ótima de frutos entre 5 e 9 FCQ na cv. 'Princesa'. Dentro desse umbral, obtevese tamanho adequado de frutos $(134,2-151,0 \mathrm{~g}) \mathrm{e}$ produção total alta $\left(21,2-25,0 \mathrm{~kg} \mathrm{pl}^{-1}\right)$, sem debilitar o vigor vegetativo da copa a curto prazo. Portanto, o raleio manual e, fundamentalmente, o raleio químico devem ser adequadamente ajustados para atingir o nível de carga de frutos dentro do umbral mencionado anteriormente. Sob 5 FCQ de carga, apresentou-se grande perda de produção, que pode afetar negativamente a rentabilidade do pomar. No entanto, com níveis de carga frutífera próximos a 9 FCQ, diminuiu a massa fresca média dos frutos pelo aumento relativo da produção de frutos pequenos e médios, e produziu-se baixo ganho na produção total $\left(\approx 4 \mathrm{~kg}\right.$.planta $\left.{ }^{-1}\right)$. Nos anos em que a expectativa de preços for favorável ou que o maior mercado for a indústria, então, a carga de frutos poderia chegar até os 9 FCQ. No entanto, é preciso avaliar o efeito desse nível de carga sobre o vigor vegetativo a longo prazo e sobre a vida útil do pomar.

TABELA 1- Efeito da carga de frutos sobre a produção total, qualidade dos frutos e intensidade da frutificação no ano seguinte, em macieira cv. Princesa.

\begin{tabular}{|c|c|c|c|c|c|c|}
\hline \multirow[b]{2}{*}{$\begin{array}{c}\text { Carga de } \\
\text { frutos (FCQ) }\end{array}$} & \multirow[b]{2}{*}{$\begin{array}{l}\text { Produção total } \\
\qquad\left(\mathrm{kg} \mathrm{p}^{-1}\right)\end{array}$} & \multicolumn{3}{|c|}{ Distribuição de diâmetros } & \multirow[b]{2}{*}{$\begin{array}{l}\text { Massa } \\
\text { fresca } \\
\text { média } \\
(\mathrm{g})\end{array}$} & \multirow[b]{2}{*}{$\begin{array}{l}\text { Intensidade da } \\
\text { frutificação (FCQ) }\end{array}$} \\
\hline & & $\begin{array}{l}\text { Frutos pequenos } \\
\qquad\left(\mathrm{kg} \mathrm{pl}^{-1}\right)\end{array}$ & $\begin{array}{l}\text { Frutos médios } \\
\qquad\left(\mathrm{kg} \mathrm{pl}^{-1}\right)\end{array}$ & $\begin{array}{l}\text { Frutos grandes } \\
\qquad\left(\mathrm{kg} \mathrm{pl}^{-1}\right)\end{array}$ & & \\
\hline 1 & 10,8 & $0,0(0,0)$ & $1,9(17,6)$ & $8,9(82,4)$ & 197,1 & $14,3 \mathrm{a}$ \\
\hline 2 & 15,3 & $0,1(0,7)$ & $4,2(27,5)$ & $11,0(71,9)$ & 177,2 & $14,6 \mathrm{a}$ \\
\hline 3 & 17,9 & $0,5(2,8)$ & $5,6(31,3)$ & $11,8(65,9)$ & 165,6 & $14,3 \mathrm{a}$ \\
\hline 4 & 19,8 & $0,9(4,5)$ & $6,6(33,3)$ & $12,3(62,1)$ & 157,4 & $13,6 \mathrm{a}$ \\
\hline 5 & 21,2 & $1,3(6,1)$ & $7,3(34,4)$ & $12,6(59,4)$ & 151 & $13,9 \mathrm{a}$ \\
\hline 6 & 22,4 & $1,7(7,6)$ & $8,0(35,7)$ & $12,7(56,7)$ & 145,8 & $13,1 \mathrm{a}$ \\
\hline 7 & 23,4 & $2,1(9,0)$ & $8,5(36,3)$ & $12,8(54,7)$ & 141,3 & $13,3 \mathrm{a}$ \\
\hline 8 & 24,3 & $2,5(10,3)$ & $8,9(36,6)$ & $12,9(53,1)$ & 137,5 & $14,2 \mathrm{a}$ \\
\hline 9 & 25 & $2,9(11,6)$ & $9,4(37,6)$ & $12,7(50,8)$ & 134,2 & $13,0 \mathrm{a}$ \\
\hline Modelo & Log. & Linear & Log. & - & Log. & MLG \\
\hline p-valor & $<0,01$ & $<0,01$ & $<0,01$ & NS & $<0,01$ & NS \\
\hline QME & 38 & 2,5 & 13,8 & 70 & 735 & 48 \\
\hline
\end{tabular}

As letras 'NS' indicam um efeito não significativo ( $>>0,05)$. FCQ: número de frutos por centímetro quadrado de superfície transversal do tronco. QME: quadrado médio do erro. Log.: modelo logarítmico. MLG: modelo linear geral. 


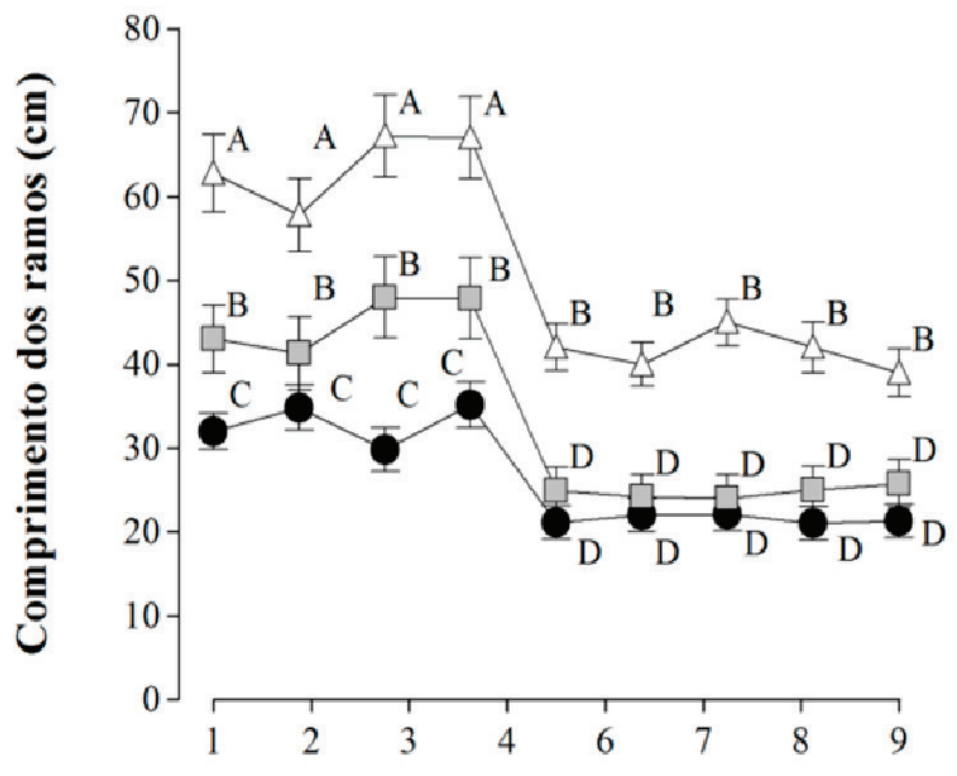

Carga de frutos (FCQ)

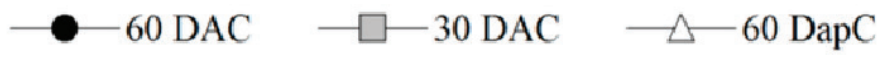

FIGURA 1- Efeito da carga de frutos sobre o comprimento dos ramos da macieira cv. 'Princesa'.

\section{REFERÊNCIAS}

ALAYÓN LUACES, P.; RODRÍGUEZ, V.A.; MARTÍNEZ, G.C. El cultivo del manzano (Malus domestica Borkh) en Corrientes: primer año de investigación. In: COMUNICACIONES CIENTÍFICAS Y TECNOLÓGICAS, 2004, Corrientes. Disponível em: $<$ http//www.unne.edu. ar/unnevieja/Web/cyt/com2004/5-Agrarias/A-029. pdf $>$. Acesso em: 20 nov. 2013.

ALBUQUERQUE JUNIOR, C. Lopes de; DENARDI, F.; DANTAS, A.C. de Mesquita; NODARI, R.O. Número de anteras por flor, grãos de pólen por antera e capacidade germinativa do pólen de diferentes cultivares de macieiras. Revista Brasileira de Fruticultura, Jaboticabal, v.32, n.4, p. 1255-1260. 2010.
CHAGAS, E.A.; CARDOSO CHAGAS, P.; PIO, R.; BETTIOL NETO, J.E.; SANCHES, J.; CARMO, S. ANTONIALI DO; CIA, P.; PASQUAL, M.; CARVALHO, A. DOS SANTOS. Produção e atributos de qualidade de cultivares de macieira nas condições subtropicais da região Leste paulista. Ciência Rural, Santa Maria, v.42, n.10, p.17641769. 2012.

DENNIS JR, F. Flowering, pollination and fruit set and development. In: FERREE, D.D.; WARRINGTON, I.J. (Ed.). Apples, botany, production and uses. Wallingford: CABI Publishing, 2003. p. 153-166.

DI RIENZO, J.A.; CASANOVES, F.; BALZARINI, M.G.; GONZALEZ, L.; TABLADA, M.; ROBLEDO, C.W. InfoStat versión 2012. Córdoba: Grupo InfoStat, FCA, Universidad Nacional de Córdoba, 2012 . Disponível em: http://www.infostat.com.ar. Acesso: 28 feb. 2012. 
DI RIENZO, J.A.; GUZMÁN, A.W.; CASANOVES, F. A Multiple Comparisons Method based on the Distribution of the Root Node Distance of a Binary Tree. Journal of Agricultural, Biological, and Environmental Statistics, New York, v.7, n.2, p 1-14. 2002

ELFVING, D.C.; SCHECHTER, I. Fruit count, fruit weight and yield relationships in 'Delicious' apple trees on nine rootstock. HortScience, Mount Vernon, v.28, n.8, p.793-795, 1993.

EMBREE, C.G.; MYRA, M.T.D.; NICHOLS, D.S.; WRIGHT, A.H. Effect of blossom density and crop load on growth, fruit quality, and return bloom in Honeycrisp' apple. HortScience, Mount Vernon, v.42, n.7, p.1622-1625, 2007.

GARCÍA, M.S. Disponibilidad de frío en la región centro de la provincia de Santa Fe. 2012. 303f. Tesis (Maestría en cultivos intensivos) - Facultad de Ciencias Agrarias. Universidad Nacional del Litoral, Santa Fe, 2012.

HAUAGGE, R.; CUMMINS, J.N. Pome fruit genetic pool for production in warm climates. In: EREZ, A. (Ed.). Temperate fruit crops in warm climates. Dordrecht: Kluwer Academic, 2000. p. 267-304.

HO, L.C. Fruit growth and sink strength. In: MARSHALL, C.; GRACE, J. (Ed.). Fruit and seed production: aspects of development, environmental physiology and ecology. Cambridge: Cambridge University Press, 1992. p.101-124.

IGLESIAS, I.; ECHEVERRÍA, G.; LOPEZ, M.L. Fruit color development, anthocyanin content, standard quality, volatile compound emissions and consumer acceptability of several 'Fuji' apple strains. Scientia Horticulturae, Amsterdam, v.137, p.138147. 2012.

IGLESIAS, I.; ECHEVERRÍA, G.; SORIA, Y. Differences in fruit color development, anthocyanin content, fruit quality and consumer acceptability of eight 'Gala' apple strains. Scientia Horticulturae, Amsterdam, v.119, p.32-40. 2008.

INGLESE, P.; CARUSO, T.; GUGLIUZZA, G.; PACE, L.S. Crop load and rootstock influence on dry matter partitioning in trees of early and late ripening peach cultivars. Journal of the American Society of Horticultural Scicence, Alexandria, v.127, n.5, p.825-830, 2002.
LINK, H.. Significance of flower and fruit thinning on fruit quality. Plant Growth Regulation, Netherlands, v.31, p.17-26. 2000.

LOPES, P.R. COELHO; OLIVEIRA, I.V. de MORAIS; SILVA, R.R. SALUSTRIANO da; CAVALCANTE, Í.H. LUCENA. Growing Princesa apples under semiarid conditions in northeastern Brazil. Acta Scientiarum: Agronomy, Maringá, v.35, n.1, p.93-99. 2013.

MARCELIS, L.F.M. Sink strength as a determinant of dry matter partitioning in the whole plant. Journal of Experimental Botany, Lancaster, v.47, p.12811291. 1996.

MEIER, U.H.; GRAF, H.; HACK, M.; HESS, W.; KENNEL, R.; KLOSE, D.; MAPPES, D.; SEIPP, R.; STAUSS, J.; STREIF J.; VAN DEN BOOM, T. Phänologische Entwick-lungsstadien des Kernobstes (Malus domestica Borkh.und Pyrus communis L.), des Steinobstes (Prunus-Arten), der Johannisbeere (Ribes-Arten) und der Erdbeere (Fragaria $x$ ananassa Duch.). Nachrichtenblatt des Deutschen Pflanzenschutzdienstes, Sttugart, v.46, p.141-153. 1994.

MELAND, M. Effects of different crop loads and thinning times on yield, fruit quality, and return to bloom in Malus $\times$ domestica Borkh. 'Elstar'. Journal of Horticultural Science \& Biotechnology ISAFRUIT, Queens Road, p.117-121, 2009. Special Issue

MENDOZA-GONZÁLEZ, S.; MARTÍNEZPENICHE, R.Á.; FERNÁNDEZ-MONTES, M.R.; RUMAYOR-FLORES, A.; CASTILLOCASTAÑEDA, E. Época de maduración y calidad del fruto de genotipos de manzana en Cadereyta, Qro. Revista Chapingo Serie Horticultura, Chapingo, v.14, n.1, p.71-78. 2008

O'ROURKE, D. World production, trade, consumption and economic outlook for apples. In: FERREE, D.D.; WARRINGTON, I.J. (Ed.). Apples: botany, production and uses. Wallingford: CABI Publishing, 2003. p.15-30.

PINHEIRO, J.; BATES, D.; DEBROY, S.; SARKAR, D. R Development Core Team. nlme: linear and nonlinear mixed effects models. $\mathrm{R}$ package version 3.1-117. 2013. Disponível em: http://CRAN.Rproject.org/package=nlme. 2 , jul. 2013. 
R DEVELOPMENT CORE TEAM. R: A language and environment for statistical computing. $R$ package version 3.0.2. R Foundation for Statistical Computing, Vienna, Austria. Disponível em: $<\underline{\mathrm{http}}: / /$ www.r-project.org/>. 2, jul. 2013.

SEIPEL, M.; PIROVANI, M.E.; GÜEMES, D.R.; GARIGLIO, N.F.; PIAGENTINI, A.M. Características fisicoquímicas de los frutos de tres variedades de manzanas cultivadas en la región centro-este de la provincia de Santa Fe. Revista FAVE - Ciencias Agrarias, Esperanza, v.8, n.1, p.27-36. 2009.

TREDER, W. Crop loading studies with 'Jonagold' apple tree. Journal of Fruit and Ornamental Plant Research, Pomologiczna, v.18, n.1, p.59-69. 2010.
TREDER, W. Relationship between yield, crop density coefficient and average fruit weight of 'Gala' apple. Journal of Fruit and Ornamental Plant Research, Pomologiczna, v.16, p.53-63, 2008.

VOLZ, R.K. Regulation and estimation of crop load on 'Gala' apple trees. New Zealand Journal of Experimental Agriculture, Wellington, v.16, n.1, p.47-53. 1988.

WRIGHT, A.H; EMBREE, C.G.; NICHOLS, D.S.; PRANGE, R.K.; HARRISON, P.A.; DELONG, J.M. Fruit mass, colour and yield of 'Honeycrisp'TM apples are influenced by manually-adjusted fruit population and tree form. Journal of Horticultural Science and Biotechnology, Queens Road, v.81, n.3, p.397-401. 2006. 